\title{
Insulin receptor-mediated signaling regulates pluripotency markers and lineage differentiation
}

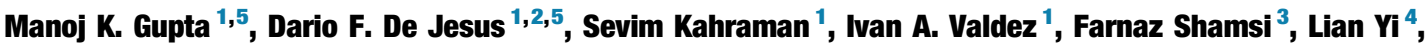 \\ Adam C. Swensen ${ }^{4}$, Yu-Hua Tseng ${ }^{3}$, Wei-Jun Qian ${ }^{4}$, Rohit N. Kulkarni ${ }^{1, *}$
}

\section{ABSTRACT}

Objectives: Insulin receptor (IR)-mediated signaling is involved in the regulation of pluripotent stem cells; however, its direct effects on regulating the maintenance of pluripotency and lineage development are not fully understood. The main objective of this study is to understand the role of IR signaling in pluripotency and lineage development.

Methods: To explore the role of IR signaling, we generated IR knock-out (IRKO) mouse induced pluripotent stem cells (miPSCs) from E14.5 mouse embryonic fibroblasts (MEFs) of global IRKO mice using a cocktail of four reprogramming factors: 0ct4, Sox2, Klf4, cMyc. We performed pluripotency characterization and directed the differentiation of control and IRKO iPSCs into neural progenitors (ectoderm), adipocyte progenitors (mesoderm), and pancreatic beta-like cells (endoderm). We mechanistically confirmed these findings via phosphoproteomics analyses of control and IRKO iPSCs.

Results: Interestingly, expression of pluripotency markers including KIf4, Lin28a, Tbx3, and cMyc were upregulated, while abundance of Oct4 and Nanog were enhanced by 4-fold and 3-fold, respectively, in IRKO iPSCs. Analyses of signaling pathways demonstrated downregulation of phospho-STAT3, p-mTor and p-Erk and an increase in the total mTor and Erk proteins in IRKO iPSCs in the basal unstimulated state. Stimulation with leukemia inhibitory factor (LIF) showed a $\sim 33 \%$ decrease of phospho-ERK in IRKO iPSCs. On the contrary, Erk phosphorylation was increased during in vitro spontaneous differentiation of iPSCs lacking IRs. Lineage-specific directed differentiation of the iPSCs revealed that cells lacking IR showed enhanced expression of neuronal lineage markers (Pax6, Tubb3, Ascl1 and Oligo2) while exhibiting a decrease in adipocyte (Fas, Acc, Ppary, Fabp4, C/ebp $\alpha$, and Fsp27) and pancreatic beta cell markers (Ngn3, Is/1, and Sox9). Further molecular characterization by phosphoproteomics confirmed the novel IR-mediated regulation of the global pluripotency network including several key proteins involved in diverse aspects of growth and embryonic development.

Conclusion: We report, for the first time to our knowledge, the phosphoproteome of insulin, IGF1, and LIF stimulation in mouse iPSCs to reveal the importance of insulin receptor signaling for the maintenance of pluripotency and lineage determination.

(c) 2018 The Authors. Published by Elsevier GmbH. This is an open access article under the CC BY-NC-ND license (http://creativecommons.org/licenses/by-nc-nd/4.0/)

Keywords Insulin receptor signaling; Pluripotency; Lineage differentiation; Adipocyte; Beta cells; Neurons; Stem cells; Phosphoproteomics; Reprogramming

\section{INTRODUCTION}

The insulin/insulin-like growth factor (IGF) family regulates the preand post-natal development and maintenance of optimum metabolic functioning of virtually all mammalian cells [1-5]. Previous studies demonstrated the importance of IGFII/IGF1R and ERBB2 receptor signaling in the maintenance of self-renewal of human embryonic stem cells (ESCs) $[6,7]$. During lineage differentiation of human pluripotent stem cells, insulin has been shown to interact with Wnt/ beta-catenin pathways redirecting mesoderm and endoderm towards neuroectoderm while inhibiting cardiac mesoderm $[8,9]$.
Reprogramming of somatic cells into pluripotent stem cells was also demonstrated to be negatively regulated by insulin growth factor pathways [10]. In mouse ESCs, the PI3K/Akt signaling pathway promotes self-renewal via IGF1R, and, while IGF signaling regulates embryonic cardiac proliferation, insulin signaling plays a role in development and metanephrogenesis [8,11-15]. Finally, IGFs have been reported to regulate vasculogenesis in pluripotent stem cells by predisposing their differentiation into mesodermal lineages [16].

In this study, we explored the direct role of insulin receptor-mediated signaling in pluripotency maintenance and in lineage development by reprogramming insulin receptor global knockout (IRKO) mouse

\footnotetext{
${ }^{1}$ Islet Cell and Regenerative Biology, Joslin Diabetes Center, Department of Medicine, Brigham and Women's Hospital, Harvard Stem Cell Institute, Harvard Medical School, Boston, MA 02215, USA ${ }^{2}$ Graduate Program in Areas of Basic and Applied Biology (GABBA), Abel Salazar Biomedical Sciences Institute, University of Porto, 5000 Porto, Portugal ${ }^{3}$ Section of Integrative Physiology and Metabolism, Joslin Diabetes Center, Harvard Medical School, Boston, MA 02215, USA ${ }^{4}$ Biological Sciences Division, Pacific Northwest National Laboratory, Richland, WA 99352, USA

5 These authors contributed equally to this work.

*Corresponding author. Islet Cell and Regenerative Biology, Joslin Diabetes Center, One Joslin Place, Boston, MA 02215, USA. Fax: +1 617 309 3476. E-mail: rohit. kulkarni@joslin.harvard.edu (R.N. Kulkarni).
} 
embryonic fibroblasts into iPSCs [17]. IRKO iPSCs demonstrated upregulation of pluripotency markers including Oct4 and Nanog. Simultaneously, key signaling pathways including Stat3/mTor/Erk were downregulated in the basal state while Erk signaling was upregulated during spontaneous differentiation into embryoid bodies. Directed differentiation analysis revealed that neuronal markers (ectoderm) were upregulated while adipocyte (mesoderm) and pancreatic beta-cell (endoderm) differentiation markers were downregulated in IRKOs. Finally, unbiased phosphoproteomics analyses revealed an involvement of insulin signaling in pluripotency, growth and development. Together, these studies underscore the importance of insulin-mediated signaling for maintenance of pluripotency and lineage development.

\section{MATERIAL AND METHODS}

\subsection{Mice and mouse embryonic fibroblasts (MEFs)}

All studies involving mice were approved by the Institutional Review Board of the Joslin Diabetes Center and were in accordance with National Institute of Health (NIH) guidelines. Embryonic day 14.5 wild type control and insulin receptor (IR) knockout (IRKO) MEFs were derived from breeding IR heterozygous mice (Jackson Laboratory Inc.). All fibroblasts were maintained up to a maximum passage $\sim \# 10$ in Dulbecco Modified Eagle's Media (DMEM) supplemented with Glutamax, 10\% Fetal Bovine Serum (FBS), and 1\% non-essential amino acids.

\subsection{Lentiviral-mediated reprogramming and IPSC generation and characterization}

Generation of mouse iPSCs involved infection of primary MEFs with mouse STEMCCA lentivirus vector expressing the reprogramming factors Oct4, Sox2, Klf4, and cMyc. iPSC characterization involved teratoma formation, H\&E staining, and immunostaining for the three lineage markers performed according to previous reports [18-20]. Briefly, MEFs $\left(5 \times 10^{4}\right)$ were plated in six well plates and virally transduced with the lentiviral particles in the presence of $5 \mu \mathrm{g} / \mathrm{ml}$ Polybrene ${ }^{\circledR}$ (EMD Millipore) after 8-24 h. The fibroblasts were washed three times with PBS and fed fresh $15 \%$ mouse embryonic stem cell (ESC) media supplemented with leukemia inhibitory factor (LIF) (EMD millipore). On days 7-14, ESC-like colonies were individually picked, cultured, expanded, frozen and subsequently characterized in a 2i-media feeder-free system for pluripotency markers. Sex determination of iPSCs was performed by using primers R05 and R03 which exclusively amplify sex-determining region of the 326 base pair of Chr Y (Sry). IRS1 amplification of the 480 base pair was used as internal control.

\subsection{Gene expression analyses using quantitative RT-PCR and western immunoblotting}

RNA extraction was performed using standard Trizol reagent (Invitrogen) according to the manufacturer's instructions; the resultant aqueous phase was mixed (1:1) with 70\% RNA-free ethanol and added to Qiagen Rneasy mini kit columns (Qiagen), and the manufacturer's protocol was followed. RNA quality and quantity were analyzed using Nanodrop 1000. One microgram of RNA was used for reserve transcription step using the highcapacity cDNA synthesis kit (Applied Biosciences) according to manufacturer instructions. cDNA was analyzed using the $A B I$ 7900HT system (Applied Biosciences), and gene expression was calculated using the $\Delta \Delta$ Ct method. Each RT-PCR was run in triplicate samples, and data was normalized to $\beta$-actin according to previous reports [21]. In parallel experiments, total cellular proteins were harvested using M-PER mammalian protein extraction reagent (Thermo Scientific) followed by western immunoblotting of proteins including 0ct4 (Santa Cruz \#Bio.sc-5279), Nanog (Cell Signaling, \#8785s), Stat3 (Santa Cruz Bio. \#sc-482), $\beta$-actin (Santa Cruz Bio. \#sc-1616), pStat3 (Cell Signaling, \#9145s), IR- $\beta$ (Cell Signaling, \#3025s), IGF1R- $\beta$ (Cell Signaling, \#9750s), pErk1/2 (Cell Signaling, \#9101s), Erk1/2 (Cell Signaling, \#9102s), pmTor (Cell Signaling, \#5536s), mTor (Cell Signaling, \#2972s), pMek (Cell Signaling, \#9121s), Mek (Cell Signaling, \#9122s), plRS-1 (Cell Signaling, \#2381s), IRS-1 (Cell Signaling, \#2390s), PI3K85 (Millipore, \# 06-496), PDK1 (Cell Signaling, \#3062s), $\alpha$-tubulin (Abcam, \#ab7291). The blots were developed using chemiluminescent substrate (ECL, ThermoFisher, MA).

\subsection{Embryoid body formation}

Control and IRKO iPSCs grown in a $2 \mathrm{i}$ system were collected using accutase (Invitrogen), and two million control or IRKO iPSCs were seeded in $10 \mathrm{~cm}$ petri-dishes containing high glucose DMEM supplemented with $20 \%$ FBS without LIF. Media were replaced every $24 \mathrm{~h}$, and cells started to form EBs at day 2 of differentiation. On days 5 and 10, EBs were harvested for transcript and signaling analyses.

\subsection{Neuronal differentiation}

Control and IRKO iPSCs grown in a $2 \mathrm{i}$ system were collected using accutase (Invitrogen). Fifty thousand control and IRKO iPSCs were plated into gelatin-coated 6-well plates and treated with differentiation media and followed for 10 days in Ndiff 227® media (Clontech) [22]. Cells were harvested on day 10 for transcript analyses of neuronal markers.

\subsection{Adipocyte differentiation}

Control and IRKO iPSCs were differentiated into adipocytes using a slightly modified protocol from Cuaranta-Monroy et al. [23]. The protocol allows iPSCs to differentiate into adipocytes in 27 days in response to a combination of cocktails at various steps (Fig. S3C). The adipocytes were subjected to oil-red 0 staining for confirmation of lipid droplets. Furthermore, total RNA was isolated for transcript analyses of adipocyte markers. We used a spontaneous method of EB production rather than the hanging drop method to enable a larger yield of EBs.

\subsection{Pancreatic beta cell differentiation}

Control and IRKO iPSCs were differentiated into pancreatic beta-like cells using a protocol from Szu-Hsui Liu et al. [24]. Pancreatic betalike cells were obtained on day 8. Total RNA was isolated from day 8 differentiated cells for transcript analyses of beta cell developmental markers. The differentiated beta-like cells were immunostained for chromogranin A (ab15160, abcam) and Ngn3 (F25A1B3, Developmental studies Hybridoma Bank, DSHB) proteins.

\subsection{Phosphoproteomics}

\subsubsection{Phosphoproteomics - Phosphopeptide enrichment}

Control and IRKO iPSCs ( $n=3 /$ group) were pelleted, washed with cold PBS, and lysed with ice-cold lysis buffer (8 M urea, $50 \mathrm{mM}$ Tris pH 8.0, $1 \mathrm{mM}$ ethylenediaminetetraacetic acid (EDTA)), $10 \mathrm{mM}$ $\mathrm{NaF}, 1: 100$ phosphatase inhibitors (Sigma)). Proteins were reduced and alkylated with $5 \mathrm{mM}$ dithiothreitol (DTT) and $10 \mathrm{mM}$ iodoacetamide followed by trypsin (Promega) digestion for $3 \mathrm{~h}$ at $37^{\circ} \mathrm{C}$. 
Peptides were subjected to clean-up via a C18 solid phase extraction column (SepPak). Phosphopeptides were enriched using Fe3+-NTA Agarose Beads (Qiagen) as previously described [25]. Enriched phosphopeptides were lyophilized and stored at $-80{ }^{\circ} \mathrm{C}$ until analyses.

\subsubsection{Global liquid chromatography tandem-mass spectrometry}

Phosphopeptide samples were dissolved in $2 \%$ acetonitrile and $0.1 \%$ formic acid immediately before being injected onto a liquid chromatography-mass spectrometry (LC-MS) system consisting of a nanoACQUITY UPLC $®$ system with a $75 \mu \mathrm{m} \times 20 \mathrm{~cm}$ C18 LC column and an Orbitrap Q-Exactive HF mass spectrometer (Thermo). A 110minute gradient was applied for LC separation. The MS was operated at a resolution of 60,000 for MS scans and 30,000 for HCD MS/ MS scans.

\subsubsection{Phosphoproteomics data analysis}

Data were analyzed at the peptide level using MaxQuant software (version 1.5.3.30) using match between runs with a false discovery rate of 0.01 . We filtered out phosphosites that had missing values in more than $80 \%$ of samples, imputed missing values with half of the minimum intensity of the phosphosites, and normalized all samples to have the same median intensity followed by $\log _{2}$-transformation. Since principal component analysis showed sample heterogeneity, we accounted for batch effects and estimated weights per sample using an unbiased algorithm that assesses how well each sample's intensity matches those of its group [26]. We compared phosphosite intensities between groups with the linear modeling package limma [27]. We also used limma to plot Venn diagrams of significant phosphosites. We plotted the heat map with the heatmap.2 function from the gplots package. We compared pathways using the limma package roast method [28]. Pathway barplots were plotted with the ggplot2 package [29]. Bioinformatics analysis was done using $\mathbf{R}$ software [30].

\section{RESULTS}

\subsection{Loss of insulin receptor (IR) in pluripotent stem cells upregulates pluripotency network}

We generated control and IRKO MEFs from day 14.5 mouse embryos of mixed genders and reprogrammed them into iPSCs using the STEMCCA plasmid (Figure S1A,B) [18]. Real-time PCR and western blot analysis confirmed almost complete absence of IR in IRKO IPSCs, while expression of IGF1R remained unchanged compared to control iPSCs (Figure 1A,B). Cell counting analyses and Ki67-staining of control and IRKO iPSCs by flow cytometry showed similar proliferation profiles and did not reveal differences between groups (Figure S1C,D). Both groups of iPSCs were morphologically similar, as shown by bright field images and alkaline phosphatase staining (Figure S1E, F). Transcript expression of pluripotency markers including Klf4, Lin28a, $T b \times 3$, and $c-M y c$ were upregulated in IRKO iPSCs (Figure 1C). Western blot and immunohistochemistry demonstrated upregulation of 0ct4 and Nanog proteins, while flow cytometry showed upregulated SSEA1, a surface pluripotency marker, in IRKO iPSCs (Figure 1D,E and S1G). Interestingly, IRKO iPSCs presented a molecular memory of increased stemness with increased Oct4, Sox2, and KIf4 mRNA after removal of LIF for $24 \mathrm{~h}$ (Figure 1F). Western blot for Oct4 and Nanog proteins showed abundance of their expression in IRKO iPSCs (Figure 1G,H). Injection of control and IRKO iPSCs into NOD SCID mice led to formation of similar sized teratomas indicating an ability to differentiate into the three lineages (Figure $\mathrm{S} 1 \mathrm{H}, \mathrm{I}$ and J). Interestingly, we observed significant downregulation of IGF1R in differentiating IRKO iPSCs in the absence of LIF for $24 \mathrm{~h}$ indicating a link between IR and IGF1R during differentiation (Figure S1K and L). These data suggest an association between loss of IR with enhanced stemness in the pluripotent stage and that IR removal is associated with a decrease in IGF1R expression during differentiation of iPSCs.

\subsection{Insulin receptor ablation impacts key pluripotency pathways}

To explore the crosstalk between proteins in the insulin signaling and pluripotency pathways, we examined iPSCs in the basal (unstimulated) versus stimulated states. In the unstimulated state, phosphorylation of Stat3 (y705), mTor (s2448), and Erk (thr202/y204) proteins were significantly decreased while total mTor and Erk proteins were upregulated in IRKO iPSCs (Figure 2A,B). Among other proteins in the insulin signaling cascade, IRS1 and the $85 \mathrm{kDa}$ subunit of PI3K were not significantly altered while PDK1 was upregulated in IRKO iPSCs (Figure S2A,B). Next, we starved iPSCs overnight followed by stimulation with insulin (100 nM), IGF1 (100 nM) or LIF (100 units/ml) for $15 \mathrm{~min}$. As expected, the phosphorylation of Akt was virtually absent or significantly reduced after insulin or IGF1 stimulation in IRKO IPSCs (Figure 2C,D).

\subsection{Erk pathway is upregulated during differentiation of IRKO} iPSCs

To examine the relevance of insulin signaling in differentiation, we undertook in vitro experiments to generate embryoid bodies (EBs) from control and IRKO iPSCs. Morphological evaluation on day 10 revealed larger EBs differentiating from IRKO iPSCs (Figure 2E,F). Furthermore, western blot analyses of unstimulated day $10 \mathrm{EBs}$ revealed significant upregulation of phospho-Erk in the IRKO group. The phosphorylation of Mek was also upregulated in IRKOs but did not reach statistical significance (Figure 2G,H). Overnight starved day 10 EBs continued to exhibit significantly elevated phospho-Erk. Upon stimulation with insulin (100 nM) a significant increase in p-Akt, p-Erk and p-Stat3 was evident in both groups, but no significant differences were observed between groups (Figure S2C and D).

\section{4. iPSCs lacking IR exhibit enhanced expression of neuronal differentiation markers}

We next undertook differentiation of the iPSCs to generate tissues from the three germ layers. To investigate the role of insulin signaling in tissues originating from the ectoderm, we directed their differentiation towards the neuronal lineage (Figure S3A, B). While the neuronal progenitor marker (Tubb3) was evident on day 10 of differentiation in both groups (Figure $3 \mathrm{~A}$ ), we observed relatively prominent neural rosettes and enhanced intensity of class III tubulin neurons in the IRKOiPSCs compared to control iPSCs (Figure 3A). Consistently, transcript levels of multiple neuronal markers including Pax6, Tubb3, Ascl1, and Oligo2 were significantly upregulated in neuronal progenitors differentiated from IRKO iPSCs as compared to controls, indicating their role in neurogenesis (Figure 3B). These results indicate that lack of IR mediated signaling prompts mouse iPSCs to differentiate towards the ectodermal lineage differentiation.

\subsection{Absence of IR in iPSCs limits differentiation towards adipocytes and pancreatic beta cells}

Next, we chose to direct the differentiation of the iPSCs towards the mesodermal lineage to address the significance of insulin signaling in adipogenesis. The iPSCs were differentiated into pre-adipocytes over 27 days using a modified protocol from Curanat-Monroy et al. [23] (Figure S3C). Morphological analyses of differentiated adipocytes at 
A

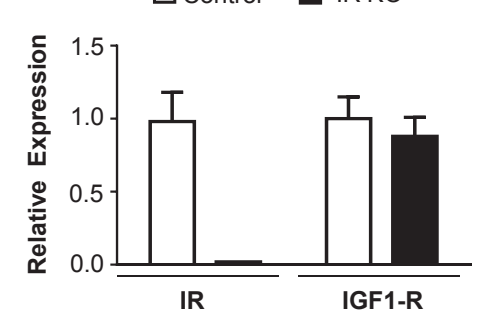

D

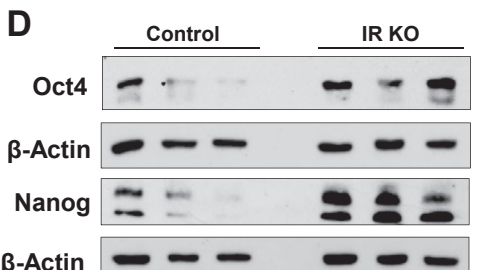

F

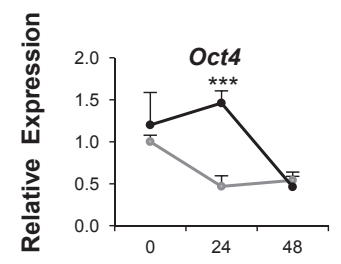

B

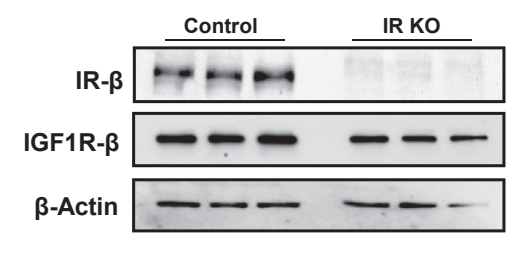

E

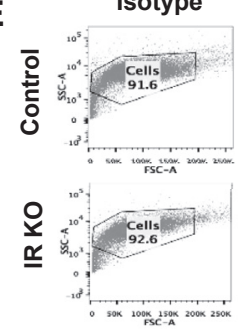

SSEA-1 FITC
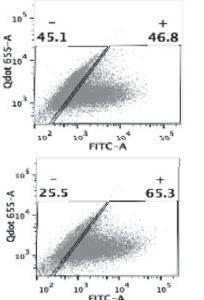

C
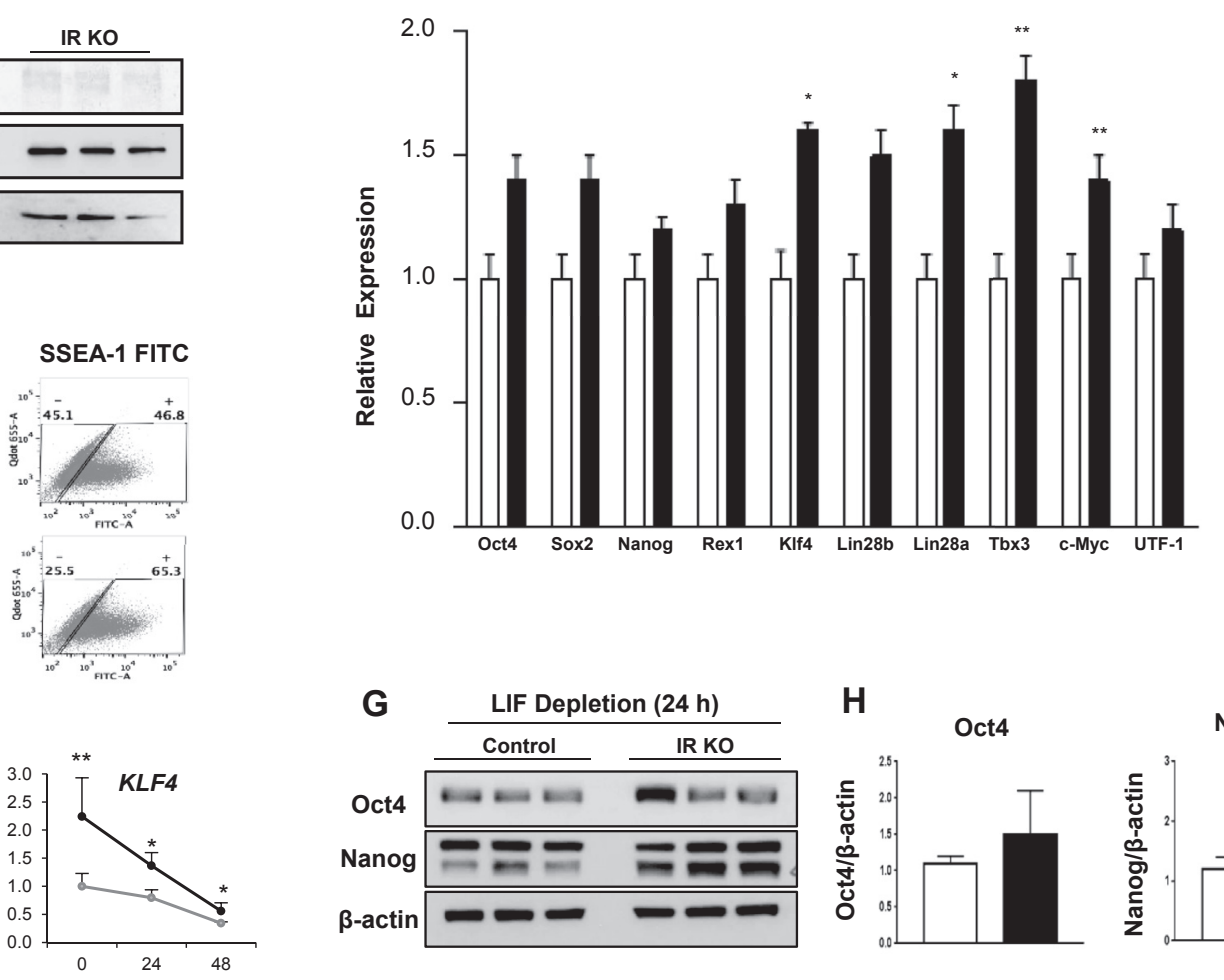

G

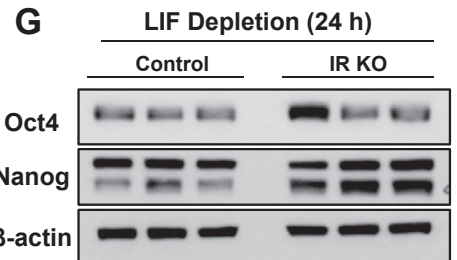

H

Oct4

Nanog

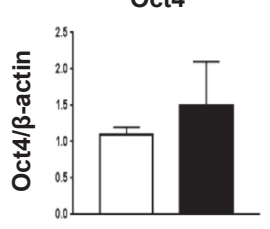

Figure 1: Mouse insulin receptor knockout (IRK0) induced pluripotent stem cells (iPSCs) revealed enhanced pluripotency network. A. Real-time PCR analysis, and B. Western blot analysis showed complete reduction of IR while IGF1-R levels were unchanged. C. Real time PCR analyses demonstrated the upregulation of core pluripotency markers in IR KO iPSCs as compared to control iPSCs. D. Western blot analysis demonstrated the significant increase of key pluripotency proteins Oct4 and Nanog in IRKO IPSCs as compared to controls. E. Flow cytometry analysis described higher expression of pluripotency surface marker, SSEA-1, in IRKO iPSCs. F. RT-PCR analysis demonstrated that key pluripotency markers Oct4, Nanog, Sox2, and KIf4 have higher expression level in IRKO iPSCs compared to Control iPSCs at $24 \mathrm{~h}$ and $48 \mathrm{~h}$ after leukemia Inhibitory factor (LIF) removal during differentiation. G. Western blot analysis showed an upregulation of Oct4 and Nanog proteins in IRKO iPSCs after $24 \mathrm{~h}$ of LIF removal. H. Quantification analysis of Oct4 and Nanog. $\beta$-actin was used as a housekeeping control. All experiments represent 3 independent experiments using 3 independent biological clones/ groups. Data are shown as mean \pm SEM. Statistical significance was determined by unpaired two-tailed student's t-test. ( $\left.{ }^{\star} \mathrm{p}<0.05,{ }^{\star \star} \mathrm{p}<0.01,{ }^{\star \star \star} \mathrm{p}<0.001\right)$. 


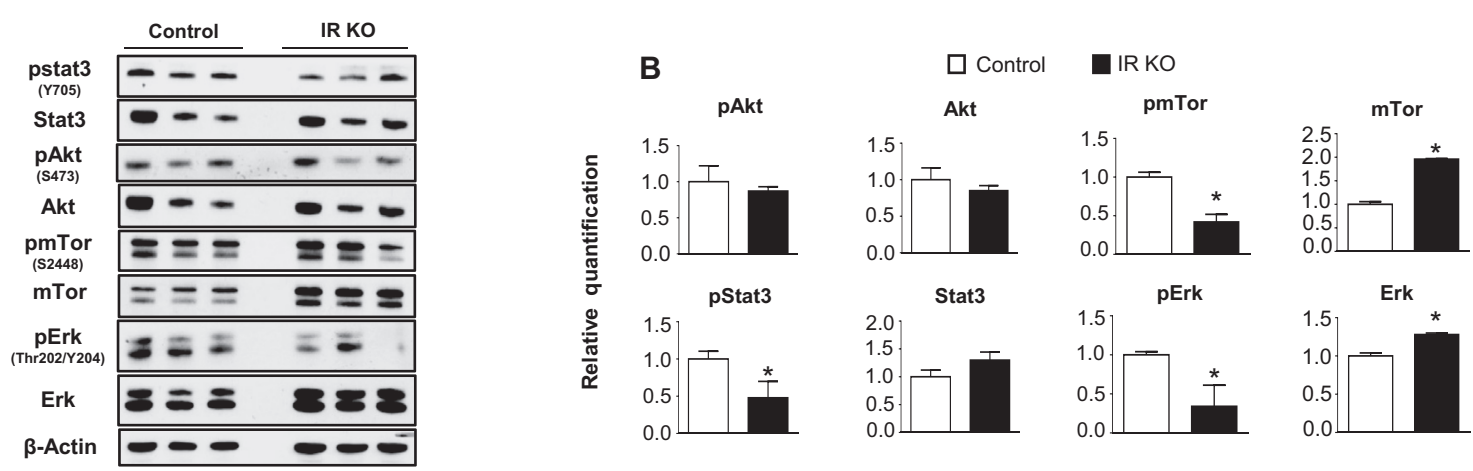

\section{C} $\frac{\text { Control }}{\text { Un INS IGF1 Lif }} \frac{\text { IR KO INS IGF1 Lif }}{}$

pStat3

Stat3

pAkt

Akt

E

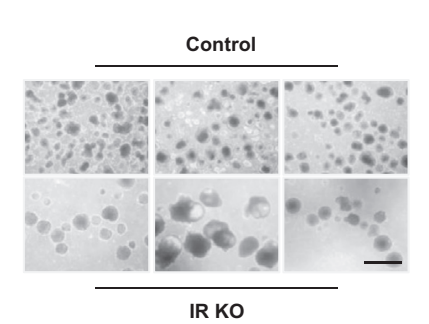

$$
\text { F }
$$

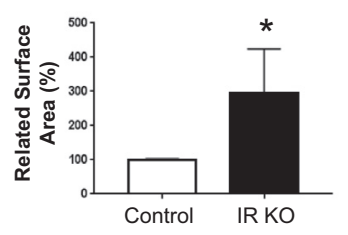

D

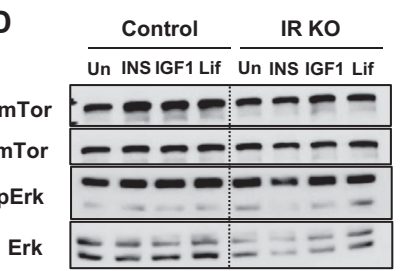

G

\begin{tabular}{|c|c|c|}
\hline \multirow[b]{2}{*}{ pErk } & Control & IR KO \\
\hline & $==$ & $===$ \\
\hline Erk & $===$ & $===$ \\
\hline$\beta$-Actin & -- & $--\cdots$ \\
\hline pMek & $-\cdots$ & $-1-$ \\
\hline Mek & $-\infty=$ & $-\infty-$ \\
\hline$\beta$-Actin & -- & $-\infty$ \\
\hline
\end{tabular}

H
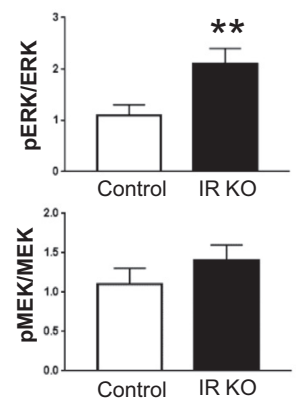

Figure 2: Signaling pathway analysis demonstrated the differential regulation of growth pathways between Control and IRKO iPSCs at basal and starved conditions. A and B. Basal signaling pathways analysis revealed no change in pAkt, Akt, and Stat3 and a decrease in pStat3, pmTor, and pErk in IRKO iPSCs. However, total proteins of mTOR and Erk were upregulated in IRKO iPSCs as compared to control iPSCs. C and D. In overnight starved conditions, IRKO iPSCs showed complete Ioss of pAkt and significant reduction in pmTor and pErk after insulin stimulation (100 nM). IGF1 stimulation (100 nM) revealed reduction in pAkt, pmTor and pErk in IRKO iPSCs. Differentiation analyses of IRKO and Control iPSCs showed differential regulation of growth pathways. E. Control and IRKO day 10 differentiated embryoid bodies (EBs). F. Quantification of cell size of embryoid bodies ( $\mathrm{N}=10$ images per group quantified). G. Western blot demonstrated upregulated phosphorylation of Erk/Mek pathways in differentiated day 10 embryoid bodies of IRKO iPSCs. H. Quantification of Erk/Mek phosphorylation. $\beta$-actin was used a housekeeping control. All experiments represent 3 independent experiments using

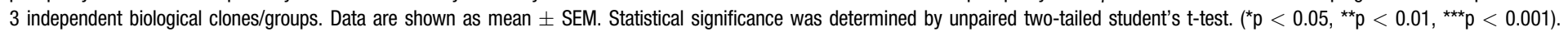


A

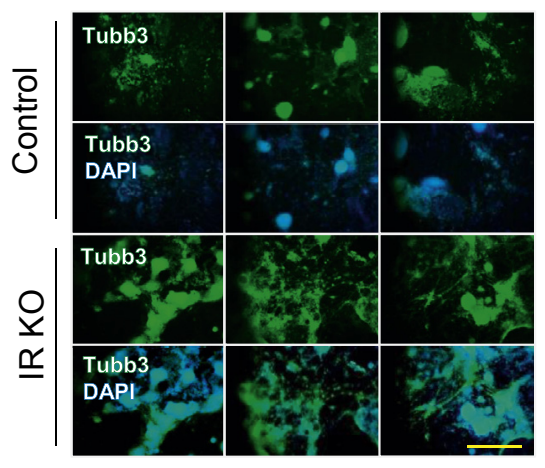

C

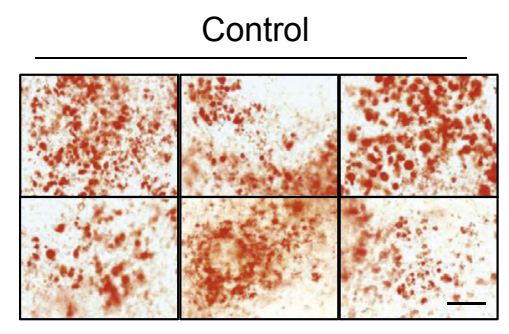

IR KO

E

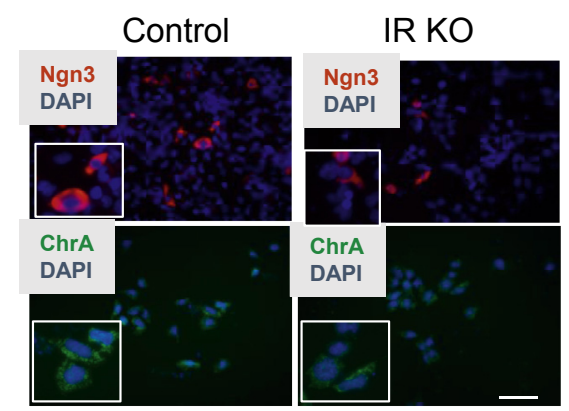

B

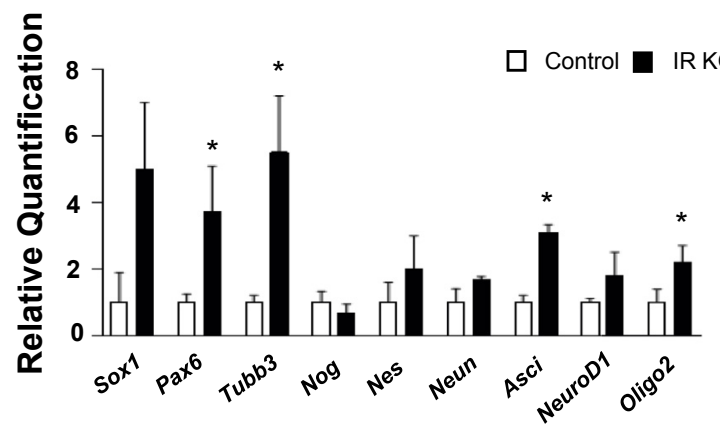

D
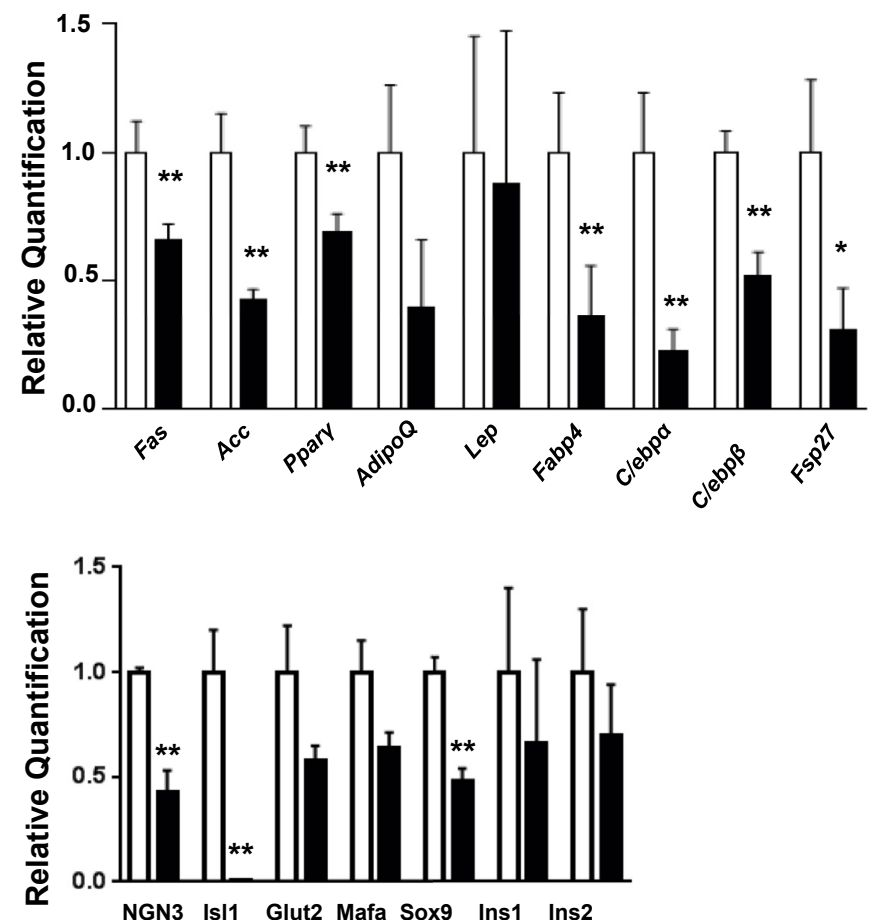

Figure 3: Upregulated neuronal and downregulated adipocyte differentiation in IRKO iPSCs. A. Beta III-tubulin immunofluorescent staining of day 10 differentiated neurons from control and IRKO iPSCs. B. RT-PCR analysis revealed that expression of neuronal markers, sex determining region Y-1 (Sox1), Paired box protein 6 (Pax6), Tubulin beta 3 class III protein (Tubb3), acid sensing ion channel (Asic), neuronal differentiation 1 (NeuroD1) and oligodendrocyte transcription factor 2 (Oligo 2) in day 10 differentiated neurons from IRKO iPSCs, while expression of other neuronal markers, noggin (Nog), Nestin (Nes) and neuronal nuclei (Neun) did not change as compared to controls. Downregulated adipocyte differentiation in IRKO iPSCs. C. Oil-red 0 staining images of day 28 adipocyte differentiation of control and IRKO iPSCs (scale bar-200um). D. RT-PCR analysis revealed downregulation of key adipocyte markers including fatty acid synthase (Fas), acetyl-coA carboxylase (AcC), fatty acid binding protein 4 (Fabp4), CCAAT/Enhancer binding protein alpha C/ebp $\alpha$, CCAAT/Enhancer binding protein beta (C/ebp $)$, peroxisome proliferator activated receptor gamma (Ppary), fat-specific protein 27 (Fsp27), while the marker of mature adipocytes leptin (Lep) did not change in IRKO iPSCs. E. Immunostaining of chromogranin A (ChrA) and neurogenein3 (Ngn3) markers in differentiated pancreatic beta cells from control and IRKO iPSCs. F. RT-PCR analyses showed downregulation of early and late beta cell differentiation markers including Ngn3, Is/1, Glut2, Mafa, Sox9, Ins1, and Ins2 in IRK0 iPSCs. All experiments represent 3 independent experiments using 3 independent biological clones/groups. Data are shown as mean \pm SEM. Statistical significance was determined by unpaired two-tailed student's t-test. ( $\left.{ }^{\star} \mathrm{p}<0.05,{ }^{* \star} \mathrm{p}<0.01,{ }^{* \star \star} \mathrm{p}<0.001\right)$. 
day 27 showed poor differentiation in adipocytes derived from IRKO iPSCs as compared to control iPSCs (Figure S3D). The limited ability to differentiate along the adipocyte lineage was supported by a reduced number of oil droplets observed in adipocytes derived from IRKO IPSCs as shown by oil-red 0 staining (Figure $3 \mathrm{C}$ ). Consistently, the expression of Fas, Acc, Ppary, Fabp4, Cebp $\alpha$, Cebp $\beta$, and Fsp27 were significantly downregulated in adipocytes differentiated from IRKO iPSCs, while the expression of AdipoQ and Leptin (Lep) was not significantly altered, probably due to the early stage of differentiation (Figure 3D). These findings are consistent with the report from Boucher et al. reporting that absence of $I R$ is detrimental for the development of adipose tissue [14].

Finally, we directed the differentiation of control and IRKO iPSCs towards the endocrine lineage (Figure S3E) using a previously published protocol [24]. Control iPSCs differentiated into endoderm-like cells at day 14 , as evidenced by bright field images of differentiated cells (Figure S3F). Immunohistochemical analyses revealed a reduction in chromogranin A+ cells and Ngn3+ cells in differentiated iPSCs deficient in IR (Figure 3E). The expression of endodermal progenitor transcripts $\mathrm{Ngn3}$, Is/1, and Sox9 were decreased significantly in IRKO iPSCs compared to controls, and genes that are typically highly expressed in mature pancreatic beta-cells, such as Glut2, Mafa, Ins1, and Ins2, presented a trend to be reduced in IRKO iPSCs (Figure 3F).

\subsection{Phosphoproteomics data reveal differentially regulated} pluripotency and development-associated proteins between control and IRKO iPSCs

We performed global phophoproteomics analyses between control and IRKO iPSCs with or without stimulation (insulin, IGF1 or LIF) and identified several differentially regulated phosphosites (Figure 4A). In the unstimulated basal state, phosphosites of several pluripotencyrelated proteins were upregulated in IRKO iPSCs compared to controls [e.g. Rif1. (S1029/2296), Kdm5b.(S1169), Slc2a3.(S482), Tfcp211.(S37), Zic3.(S203), Utf.(S99) and Tbx3.(S432)]. Similarly, phosphosites of proteins involved in embryonic development (Pwp2 (S895), Npat (T205), Kmt2d (S1562), Zscan4d (S312), Lig1 (S94)), neural development (Fxr2 (S452), Sema4b (S482)) cancer (Tmx2 (S211), Npat (T205)), and DNA damage (Mdc1 (T1113)) were all upregulated in iPSCs devoid of IR. A decreased phosphorylation was associated with proteins involved in development (Smg9(S53), Sec61b (S13), Thrap3 (S243)), mRNA splicing (Cpsf1(S754), Srm2 (S2638)), and cancer (Sash1 (S831), Prkd3 (S41), Hdac1(S410)) in IRK0 iPSCs (Figure 4B and S4A). Notably, proteins presenting increased phosphorylation in the unstimulated state in IRKO compared to control iPSCs were enriched for G0 terms associated with histone methylation, development, and telomere maintenance (Figure 4C) while downregulated phosphosites were enriched for GOs related to development, stem cell division, and autophagy (Figure 4D).

To further explore the differential regulation of proteins when insulin receptor signaling is compromised, we challenged control and IRKO iPSCs independently with insulin (100 nM), IGF1 (100 nM), or LIF (100 units/ml). Exogenous insulin regulated phosphoproteins such as Sema4b (S816), Med19 (S226), Hdac1 (S409), Gtf2f1 (T384) and Smg9 (S53). IGF1 stimulation altered Tbc1d10b (642), Ubr4 (S2715), Glis2 (S54), and Mdc1 (S1052). And, finally, LIF regulated phosphoproteins including Pias2 (S499), Fam193a (S293), Yap1 (S112), Zc3h13 (S207), Znf513 (S253), and Plekha7 (S116) (Figure 4B and S4B,C,D). Insulin regulated pathways between two groups involved in receptor binding, nutrient sensing, proliferation, development and differentiation (Figure 4E,F), while IGF1 regulated pathways related to metabolic processes, transduction, transport, membrane biology, transcription, and proteolysis
(Figure 4G,H, S4C,E). Phosphoproteomics analysis between control and IRKO iPSCs revealed proteins in the LIF regulated pathways which are involved in metabolism, development, differentiation, histone methylation, kinase activity, cell maturation and tissue development (Figure 4I,J, S4D,E). Finally, all stimuli (e.g. insulin, IGF1 or LIF) regulated phosphoproteins related to DNA damage, gene expression, cell growth, development and cancer. Interestingly, the phosphosites Thrap3 (S243), Arhgef7 (S228), Hdac1 (S410), Cpsf1 (S754), and Zgpat (S64) were regulated by all three stimuli.

To exclude the possibility that the observed changes are due to differences in total proteins, we first re-analyzed the datasets by plotting the delta delta changes (e.g. for insulin stimulation: KO vs WT with insulin and KO vs WT with no stimulation) in each of the different stimulation conditions (Figure 4B). Second, we measured the total protein levels of three candidates showing decreased phosphorylation in the unstimulated state, namely, Trim28 (Figure 4B), Sec61B (Figure S4A) and Thrap3 (Figue. 4B and S4A), in an aliquot of the lysates also submitted for phosphoproteomics. The increased total protein expression patterns for all 3 candidates in IRKO iPSCs compared to controls suggested that alterations at the phosphorylation level was independent of changes in total protein levels (Figure S4G, H). The present study uncovers previously unidentified roles for insulin receptor-mediated signaling in the regulation of proteins involved in pluripotency and lineage development. Mechanistic studies to examine the function of these novel phosphosites require further investigation.

\section{DISCUSSION}

The proteins in the insulin/IGF-1 signaling family regulate the growth and function of most mammalian cells. The role of IGFII/IGF1R in pluripotency and differentiation has been reported previously. For example, Bendall et al. reported that blocking of IGFII or loss of IGF1R cause differentiation of human pluripotent stem cells [6]. Similarly, Wang et al. also revealed that blocking of IGF1R by an antibody or lentivirus shRNA prompts human pluripotent stem cells to apoptosis and begin to differentiate [7]. Delta40p53, a transactivation deficient isoform of tumor suppressor p53, has been reported to control switching between pluripotency and differentiation by regulating the level of IGF1R/PI3K in mouse pluripotent stem cells [31].

To address the direct role of IR in the maintenance of pluripotency and lineage determination, we undertook directed differentiation of control and IRKO iPSCs obtained from day 14.5 MEFs. In the present study, we report, for the first time to our knowledge, that loss of IR leads to upregulation of key pluripotency genes involved in self-renewal and differentiation such as Oct4, Nanog, along with other markers such as Sox2, Lin28b, UTF1, Tbx3 and Rex1. Oct4, Sox2, and Nanog, all of which are key pluripotency genes involved in reprogramming, selfrenewal and differentiation [17,32]. Oct4 overexpression triggers primitive endoderm or mesoderm while 0ct4 reduction leads to trophectoderm differentiation in mouse pluripotent stem cells [33]. Wang et al. reported that Nanog represses an ectoderm differentiation program while Sox2 and Sox3 are redundant and block meso-endoderm differentiation [34]. Considering that Lin28a has been reported to control insulin/PI3K signaling via the repression of let-7 [35], it is possible that increased expression of the pluripotency network counteracts loss of IR to maintain pluripotency and self-renewal of the stem cells. Interestingly, pluripotency markers remain upregulated both at the transcript and protein levels even after removal of LIF, a key mouse pluripotency cytokine, for $24 \mathrm{~h}$ and $48 \mathrm{~h}$ in IRKO iPSCs. These findings provide new insights linking IR with regulation of pluripotency markers and differentiation. 


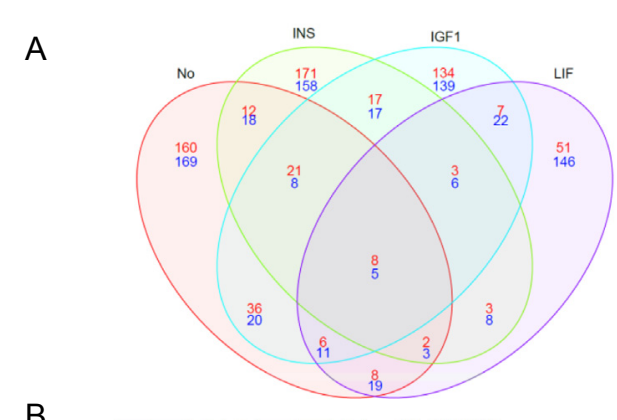

B

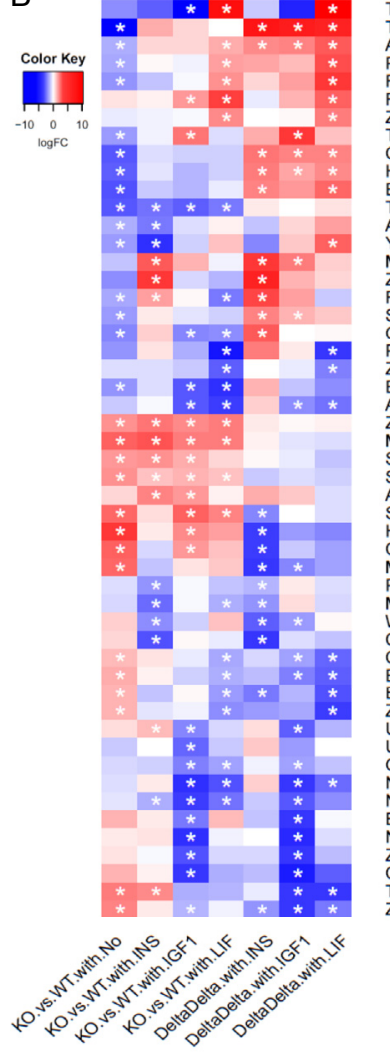

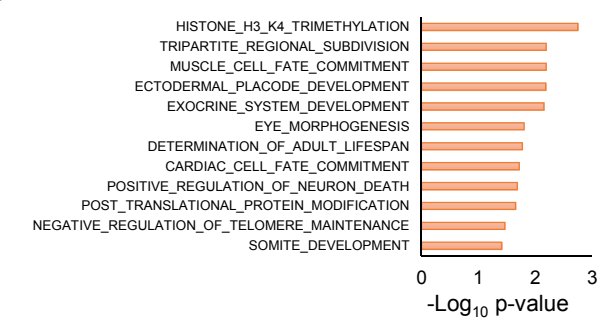

E
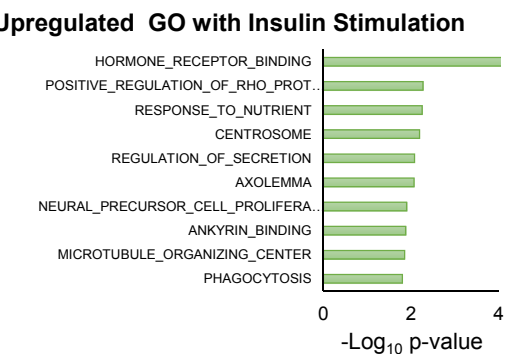

D

Downregulated GO with NO Stimulation

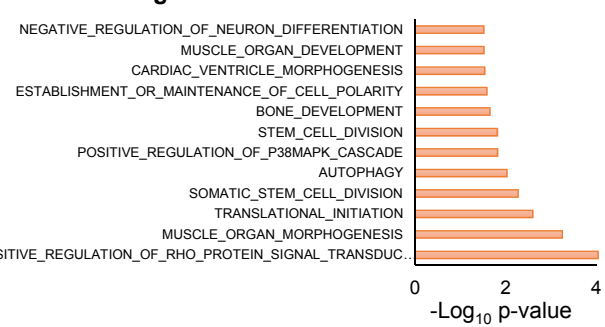

F

\section{Downregulated GO with Insulin Stimulation}

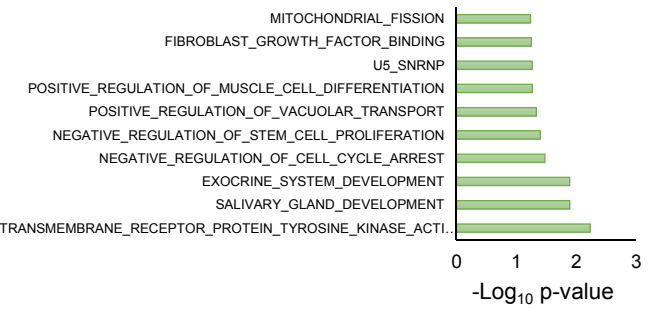

G Upregulated GO with IGF1 Stimulation

$\mathrm{H}$ Downregulated GO with IGF1 Stimu

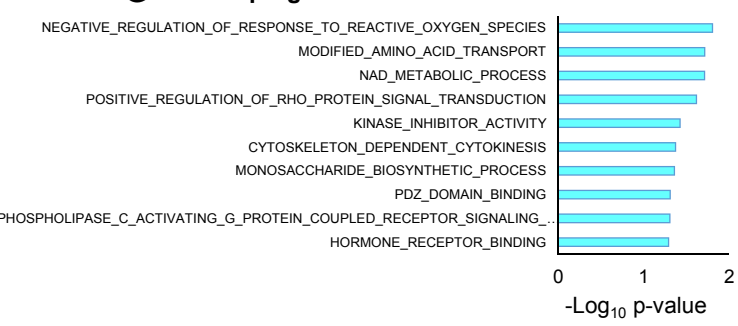

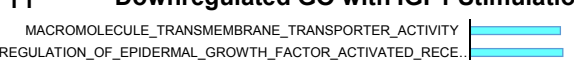

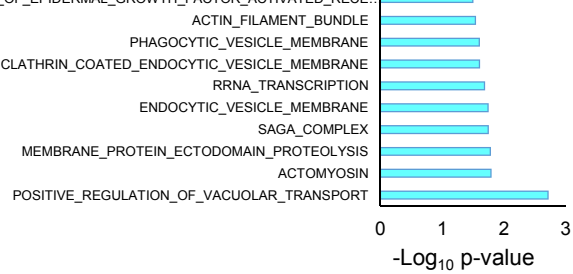

I Upregulated GO with LIF Stimulation NAD_METABOLLCPROCESS HISTONE_H3_DEACETYLATION

NEUROMUSCULAR_JUNCTIONDDEVELOPMEN

HEMATOPOEIETIC_STEM_CELL_DIFERENTIATION OXIDATIVE_DEMETHYLATION

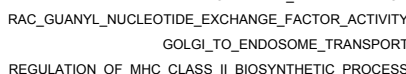
REGULATION_OF_MHC_COAST POSITIVE_REGULATION_OF_PRL_MIRNA_TRANSCRRPTION_FROM_RNA_POLYM.

Figure 4: Phosphoproteomics analyses of control and IRKO iPSCs at basal and stimulated conditions. A. Venn diagram representation of phosphoproteins differentially regulated between control and IRKO iPSCs at basal state and after 15 min stimulation with insulin (100 nM), IGF1 (100 nm) or LIF (100 units/ml). Red color denotes basal condition regulated phosphoproteins while green, blue and purple colors represent insulin, IGF1 or LIF regulated phosphoproteins. B. Heat map representation of phosphoproteins differentially regulated between control and IRKO IPSCS at basal condition and various simulated conditions including INS, IGF1 or LIF. Heat map is in centered log2 value. Red color denotes upregulated while blue color defines downregulated phosphoproteins. Delta delta changes (KO vs. WT at stimulated condition and KO vs. WT at basal) represents the unique phosphoproteins differentially regulated by stimulation with insulin, IGF1, or LIF. C and D. GO pathway analyses showing the differentially regulated pathways in red color bar graphs between IRKO vs control iPSCs at basal condition. E and F. GO pathways analyses showing differentially regulated pathways in green color bar graphs between two groups in response to insulin stimulation $\mathbf{G}$ and $\mathbf{H}$. GO pathways analyses showing differentially regulated pathways in blue color bar graphs between two groups in response to IGF1 stimulation. I and J. G0 pathways analyses showing differentially regulated pathways in purple color bar graphs between IR KO vs Control iPSCs in response to LIF stimulation. In all bar graphs, the Y-axis denotes regulated pathways, while the X-axis represents their log10 p-value. All experiments represent 3 independent experiments using 3 independent biological clones/groups. 
Next, we explored the signaling pathways involved in pluripotency and differentiation of stem cells. Stat3 and Akt pathways, along with Erk and mTor signaling, are reported to maintain growth, differentiation, and pluripotency of human and mouse pluripotent cells $[36,37]$. Interestingly, in our study, we observed a reduction in the Stat3/Erk/mTor pathways in IRKO iPSCs at basal state while the Akt pathway was virtually completely blunted after starved cells were stimulated with insulin, thus confirming the relevance of IR-mediated Akt signaling in normal pluripotent stem cells. These findings also suggest that insulin mediated Akt signaling is unlikely a critical element and can be compensated for by other pluripotency pathways. Erk pathway is involved in differentiation and was recently reported to be linked to self-renewal of mouse pluripotent stem cells [38]. Observations of decreased phosphorylation of Stat3, Akt and Erk in IRKO iPSCs led us to speculate that unidentified signaling pathways independent of IR are involved in upregulation of the pluripotency network.

While the Erk pathway has various functions involved in pluripotency of human pluripotent stem cells, in mice, Erk signaling has been linked to both pluripotency and differentiation of stem cells [38]. Trappmann et al. reported that enhanced phospho-Erk signaling reduces stem cell differentiation [39]. We observed an upregulation of Erk pathways in differentiating embryoid bodies developed from IRKO iPSCs that may have contributed to the larger EBs in this group. A more detailed study is required to investigate the role of the Erk pathway during differentiation. While genetic insulin resistance has been reported as a modulator of gene expression in human pluripotent stem cells [40], the role of insulin receptor-mediated signaling in lineage development has been studied in different contexts. For example, insulin receptor substrate 1 (IRS-1) has been reported to play a role in maintaining mouse pluripotency [41]. It has been previously described that insulinmediated signaling favors differentiation of human pluripotent stem cells into the neuroectodermal lineage at the expense of mesendodermal lineages [8]. Lian et al. reported that exogenous insulin inhibits cardiac mesoderm which can be rescued by modulation of the canonical Wnt signaling pathway [9].

To investigate the role of increased Erk pathway and loss of IR during differentiation, we directly differentiated control and IRKO iPSCs into neuronal cells (ectoderm), adipocytes (mesodermal) or beta-like cell (endoderm). IRKO iPSCs showed an upregulation of neuronal markers including early neural progenitor markers such as Pax6, Tubb3, Oligo2 and Ascl1. This is consistent with the report that the insulin receptor is involved in development of the peripheral nervous system in drosophila [42]. On the contrary, adipocytes differentiated from IRKO iPSCs presented features of reduced adipogenesis. Thus, we observed significantly reduced expression of adipocyte and lipogenesis markers, such as Fas, Acc, Fsp27, Fabp4, Cebp $\alpha$, and $C e b p \beta$. Fas and Acc are lipogenic enzymes, while Fabp4 is a lipid transporter, which is highly expressed in mature white adipocytes. Fsp27 is involved in unilocular lipid droplet and adipocyte formation [43]. Furthermore, Cebp is reported to play a developmental role in adipogenesis [44]. These data are supported by an earlier report that adipocyte-specific IRKO mice exhibit a significant $(\sim 90 \%)$ reduction in white adipocytes [14].

Finally, we explored the ability of IRKO iPSCs to differentiate towards the endocrine lineage. Among the markers that are known to contribute to pancreatic cell development, it was notable that Ngn3, Sox9, and Is/1 were all significantly downregulated, while insulin1 (Ins1) and insulin2 (Ins2) were unaltered in differentiated cells from IRKO iPSCs. These results suggest that insulin receptor-mediated signaling regulates a specific set of pancreatic cell developmental markers and warrants further investigation of the pathway during early developmental stages in mammals.

The lack of previous reports on detailed phosphoprotemics analyses underscores the importance of our observations that insulin receptor signaling regulates the pluripotency network in iPSCs and provides several novel and uncharacterized post-translational modifications in proteins involved in diverse aspects of growth, pluripotency, cell cycle and life span regulation. For instance, among the significantly altered proteins, Rif1 is reported to maintain telomere length homeostasis in pluripotent stem cells by mediating heterochromatin silencing [45]; and $\mathrm{Kdm} 5 \mathrm{~b}$ regulates self-renewal of embryonic stem cells and opposes cryptic intragenic transcription [46]. Tfcp2/1 is a transcription factor acting at the intersection of LIF and 2i-mediated self-renewal pathways to maintain ESC identity by promoting Nanog expression [47]. UTF1, Tbx3 and Zic3 which were identified in our phosphoproteomics analyses are known to be involved in pluripotency of iPSCs. Phosphoproteomics data also provided mechanistic insights into the differentiation properties of IRKO IPSCs. Thus, phosphoproteins involved in neuronal development, such as Fxr2 and Sema4b, were among those upregulated in IRKO iPSCs, confirming our findings of upregulation of neuronal differentiation markers in IRKO iPSCs. Among the several proteins involved in development and differentiation is Pwp2, which is upregulated in IRK0 iPSCs. Pwp1, a family member of Pwp2, is required for the differentiation of mouse pluripotent stem cells [48]. On the contrary, Sec61b which is required for the development of drosophila, Smg9, involved in the development of brain, heart and eye, and Thrap3, reported to play a role in bone and adipocyte development, are downregulated in IRKO iPSCs [49-51]. Taken together, our studies on directed differentiation and phophoproteomics analyses support our hypothesis that insulin receptor signaling is a key regulator of stemness and is important for the regulation of pluripotency markers and normal embryonic development. It would be interesting to contrast these data with a iPS model lacking IGF-1 receptors or both.

\section{CONCLUSION}

In summary, the present study provides a novel role for IR-mediated signaling in the global regulation of the pluripotency network and differentiation potential of ectoderm, mesoderm and endoderm lineages by upregulating the Erk pathway. The identification of several previously uncharacterized phosphosites provides a unique opportunity to further examine their significance as pluripotency regulators and development mediators.

\section{AUTHOR CONTRIBUTIONS}

Conceptualization, M.K.G., R.N.K., D.F.J.; Validation and Investigation, M.K.G., D.F.J., I.A.V.,F.S., and S.K.; Phosphoproteomics analyses, L.Y., A.C.W., W.Q.; Writing-original draft, review and editing, M.K.G., D.F.J., and R.N.K.; Supervision, R.N.K.; Funding acquisition and final approval, R.N.K. All authors have reviewed the manuscript.

\section{ACKNOWLEDGMENTS}

We thank G. Mostoslavsky PhD (Boston University) for the kind gift of STEMCCA lentiviral plasmids. We thank J.Hu for technical assistance. Authors acknowledge Joslin Diabetes Center iPS Core Facility (DRC, NIH DK036836). MKG is supported by JDRF advanced postdoctoral fellowship grant 3-APF-2017-393-A-N. DFJ was supported by the Portuguese Foundation for Science and Technology - FCT (SFRH/BD/51699/2011), Albert Ronald Travel Fellowship 2015, 2016 FLAD R\&D@PhD Internship Grant. RNK is supported by the HSCl and NIH grants R01 DK67536 and R01 DK103215. WQ is 
supported by NIH grants UC4 DK104167 and DP3 DK110844. Y-HT is supported by NIH grants R01DK077097 and R01DK102898. FS is supported by American Diabetes Association Fellowship \#1-18-PDF-169. Part of the experimental work was performed in the Environmental Molecular Sciences Laboratory, a national scientific user facility sponsored by the DOE and located at Pacific Northwest National Laboratory, which is operated by Battelle Memorial Institute for the DOE under Contract DE-AC05-76RL0 1830. All authors declare no financial conflict of interest.

\section{CONFLICT OF INTEREST}

All authors declare no conflict of interest.

\section{APPENDIX A. SUPPLEMENTARY DATA}

Supplementary data to this article can be found online at https://doi.org/10.1016/j. molmet.2018.09.003.

\section{REFERENCES}

[1] Michael, M.D., Kulkarni, R.N., Postic, C., Previs, S.F., Shulman, G.I., Magnuson, M.A., etal., 2000. Loss of insulin signaling in hepatocytes leads to severe insulin resistance and progressive hepatic dysfunction. Molecular Cell 6(1):87-97.

[2] Kulkarni, R.N., Brüning, J.C., Winnay, J.N., Postic, C., Magnuson, M.A., Kahn, C.R., 1999. Tissue-specific knockout of the insulin receptor in pancreatic B cells creates an insulin secretory defect similar to that in type 2 diabetes. Cell 96(3):329-339.

[3] Kulkarni, R.N., 2002. Receptors for insulin and insulin-like growth factor-1 and insulin receptor substrate-1 mediate pathways that regulate islet function. Biochemical Society Transactions 30:317-322.

[4] Nakae, J., Kido, Y., Accili, D., 2001. Distinct and overlapping functions of insulin and IGF-I receptors. Endocrine Reviews 22(6):818-835.

[5] Taniguchi, C.M., Emanuelli, B., Kahn, C.R., 2006. Critical nodes in signalling pathways: insights into insulin action. Nature Reviews Molecular Cell Biology 7(2):85-96.

[6] Bendall, S.C., Stewart, M.H., Menendez, P., George, D., Vijayaragavan, K., Werbowetski-Ogilvie, T., et al., 2007. IGF and FGF cooperatively establish the regulatory stem cell niche of pluripotent human cells in vitro. Nature 448(7157):1015-1021.

[7] Wang, L., Schulz, T.C., Sherrer, E.S., Dauphin, D.S., Shin, S., Nelson, A.M., et al., 2007. Self-renewal of human embryonic stem cells requires insulin-like growth factor-1 receptor and ERBB2 receptor signaling. Blood 110(12):4111-4119.

[8] Freund, C., Ward-van Oostwaard, D., Monshouwer-Kloots, J., van den Brink, S., van Rooijen, M., Xu, X., et al., 2008. Insulin redirects differentiation from cardiogenic mesoderm and endoderm to neuroectoderm in differentiating human embryonic stem cells. Stem Cells 26(3):724-733.

[9] Lian, X., Zhang, J., Zhu, K., Kamp, T.J., Palecek, S.P., 2013. Insulin inhibits cardiac mesoderm, not mesendoderm, formation during cardiac differentiation of human pluripotent stem cells and modulation of canonical Wnt signaling can rescue this inhibition. Stem Cells 31(3):447-457.

[10] Tran, K.A., Jackson, S.A., Olufs, Z.P., Zaidan, N.Z., Leng, N., Kendziorski, C., et al., 2015. Collaborative rewiring of the pluripotency network by chromatin and signalling modulating pathways. Nature Communications 6:6188.

[11] Liu, Z.Z., Kumar, A., Ota, K., Wallner, E.I., Kanwar, Y.S., 1997. Developmental regulation and the role of insulin and insulin receptor in metanephrogenesis. Proceedings of the National Academy of Sciences of the U S A 94(13):6758-6763.

[12] Li, Y., Geng, Y.J., 2010. A potential role for insulin-like growth factor signaling in induction of pluripotent stem cell formation. Growth Hormone \& IGF Research 20(6):391-398.

[13] Li, P., Cavallero, S., Gu, Y., Chen, T.H., Hughes, J., Hassan, A.B., et al., 2011. IGF signaling directs ventricular cardiomyocyte proliferation during embryonic heart development. Development 138(9):1795-1805.
[14] Boucher, J., Mori, M.A., Lee, K.Y., Smyth, G., Liew, C.W., Macotela, Y., et al., 2012. Impaired thermogenesis and adipose tissue development in mice with fat-specific disruption of insulin and IGF-1 signalling. Nature Communications 3:902.

[15] Magner, N.L., Jung, Y., Wu, J., Nolta, J.A., Zern, M.A., Zhou, P., 2013. Insulin and IGFs enhance hepatocyte differentiation from human embryonic stem cells via the PI3K/AKT pathway. Stem Cells 31(10):2095-2103.

[16] Piecewicz, S.M., Pandey, A., Roy, B., Xiang, S.H., Zetter, B.R., Sengupta, S., 2012. Insulin-like growth factors promote vasculogenesis in embryonic stem cells. PLoS One 7(2) e32191.

[17] Takahashi, K., Yamanaka, S., 2006. Induction of pluripotent stem cells from mouse embryonic and adult fibroblast cultures by defined factors. Cell 126(4): 663-676.

[18] Sommer, C.A., Stadtfeld, M., Murphy, G.J., Hochedlinger, K., Kotton, D.N., Mostoslavsky, G., 2009. Induced pluripotent stem cell generation using a single lentiviral stem cell cassette. Stem Cells 27(3):543-549.

[19] Bhatt, S., Gupta, M.K., Khamaisi, M., Martinez, R., Gritsenko, M.A., Wagner, B.K., et al., 2015. Preserved DNA damage checkpoint pathway protects against complications in long-standing type 1 diabetes. Cell Metabolism 22(2):239-252.

[20] Teo, A.K., Windmueller, R., Johansson, B.B., Dirice, E., Njolstad, P.R., Tjora, E., et al., 2013. Derivation of human induced pluripotent stem cells from patients with maturity onset diabetes of the young. Journal of Biological Chemistry 288(8):5353-5356.

[21] Gupta, M.K., Teo, A.K., Rao, T.N., Bhatt, S., Kleinridders, A., Shirakawa, J., et al., 2015. Excessive cellular proliferation negatively impacts reprogramming efficiency of human fibroblasts. Stem Cells Translational Medicine 4(10): $1101-1108$.

[22] Ying, Q.L., Stavridis, M., Griffiths, D., Li, M., Smith, A., 2003. Conversion of embryonic stem cells into neuroectodermal precursors in adherent monoculture. Nature Biotechnology 21(2):183-186.

[23] Cuaranta-Monroy, I., Simandi, Z., Kolostyak, Z., Doan-Xuan, Q.M., Poliska, S., Horvath, A., et al., 2014. Highly efficient differentiation of embryonic stem cells into adipocytes by ascorbic acid. Stem Cell Research 13(1):88-97.

[24] Liu, S.H., Lee, L.T., 2012. Efficient differentiation of mouse embryonic stem cells into insulin-producing cells. Experimental Diabetes Research 2012: 201295

[25] Mertins, P., Yang, F., Liu, T., Mani, D.R., Petyuk, V.A., Gillette, M.A., et al., 2014. Ischemia in tumors induces early and sustained phosphorylation changes in stress kinase pathways but does not affect global protein levels. Molecular \& Cellular Proteomics 13(7):1690-1704.

[26] Ritchie, M.E., Diyagama, D., Neilson, J., van Laar, R., Dobrovic, A., Holloway, A., et al., 2006. Empirical array quality weights in the analysis of microarray data. BMC Bioinformatics 7(1).

[27] Ritchie, M.E., Phipson, B., Wu, D., Hu, Y., Law, C.W., Shi, W., et al., 2015. Limma powers differential expression analyses for RNA-sequencing and microarray studies. Nucleic Acids Research 43(7) gkv007-e47.

[28] Wu, D., Lim, E., Vaillant, F., Asselin-Labat, M.L., Visvader, J.E., Smyth, G.K., 2010. ROAST: rotation gene set tests for complex microarray experiments. Bioinformatics (Oxford, England) 26(17):2176-2182.

[29] Wickham, H., 2010. A layered grammar of graphics. Journal of Computational \& Graphical Statistics 19(1):3-28.

[30] Gentleman, R.C., Carey, V.J., Bates, D.M., Bolstad, B., Dettling, M., Dudoit, S., et al., 2004. Bioconductor: open software development for computational biology and bioinformatics. Genome Biology 5(10):1-16.

[31] Ungewitter, E., Scrable, H., 2010. Delta40p53 controls the switch from pluripotency to differentiation by regulating IGF signaling in ESCs. Genes \& Development 24(21):2408-2419.

[32] Wernig, M., Meissner, A., Foreman, R., Brambrink, T., Ku, M., Hochedlinger, K., et al., 2007. In vitro reprogramming of fibroblasts into a pluripotent ES-cell-like state. Nature 448(7151):318-324. 
[33] Niwa, H., Miyazaki, J., Smith, A.G., 2000. Quantitative expression of Oct-3/4 defines differentiation, dedifferentiation or self-renewal of ES cells. Nature Genetics 24(4):372-376.

[34] Wang, Z., Oron, E., Nelson, B., Razis, S., Ivanova, N., 2012. Distinct lineage specification roles for NANOG, OCT4, and SOX2 in human embryonic stem cells. Cell Stem Cell 10(4):440-454.

[35] Zhu, H., Shyh-Chang, N., Segrè, A.V., Shinoda, G., Shah, S.P., Einhorn, W.S., etal., 2011. The Lin28/let-7 axis regulates glucose metabolism. Cell 147(1):81-94.

[36] Li, J., Wang, G., Wang, C., Zhao, Y., Zhang, H., Tan, Z., et al., 2007. MEK/ERK signaling contributes to the maintenance of human embryonic stem cell selfrenewal. Differentiation 75(4):299-307.

[37] Cherepkova, M.Y., Sineva, G.S., Pospelov, V.A., 2016. Leukemia inhibitory factor (LIF) withdrawal activates mTOR signaling pathway in mouse embryonic stem cells through the MEK/ERK/TSC2 pathway. Cell Death \& Disease 7:e2050.

[38] Chen, H., Guo, R., Zhang, Q., Guo, H., Yang, M., Wu, Z., et al., 2015. Erk signaling is indispensable for genomic stability and self-renewal of mouse embryonic stem cells. Proceedings of the National Academy of Sciences of the U S A 112(44):E5936-E5943.

[39] Trappmann, B., Gautrot, J.E., Connelly, J.T., Strange, D.G., Li, Y., Oyen, M.L., et al., 2012. Extracellular-matrix tethering regulates stem-cell fate. Nature Materials 11(7):642-649.

[40] Iovino, S., Burkart, A.M., Kriauciunas, K., Warren, L., Hughes, K.J., Molla, M., et al., 2014. Genetic insulin resistance is a potent regulator of gene expression and proliferation in human iPS cells. Diabetes 63(12):4130-4142.

[41] Rubin, R., Arzumanyan, A., Soliera, A.R., Ross, B., Peruzzi, F., Prisco, M., 2007. Insulin receptor substrate (IRS)-1 regulates murine embryonic stem (mES) cells self-renewal. Journal of Cellular Physiology 213(2):445-453.

[42] Dutriaux, A., Godart, A., Brachet, A., Silber, J., 2013. The insulin receptor is required for the development of the Drosophila peripheral nervous system. PLoS One 8(9) e71857.
[43] Zhou, L., Park, S.Y., Xu, L., Xia, X., Ye, J., Su, L., et al., 2015. Insulin resistance and white adipose tissue inflammation are uncoupled in energetically challenged Fsp27-deficient mice. Nature Communications 6:5949.

[44] Darlington, G.J., Ross, S.E., MacDougald, 0.A., 1998. The role of C/EBP genes in adipocyte differentiation. Journal of Biological Chemistry 273(46):30057-30060.

[45] Dan, J., Liu, Y., Liu, N., Chiourea, M., Okuka, M., Wu, T., et al., 2014. Rif1 maintains telomere length homeostasis of ESCs by mediating heterochromatin silencing. Developmental Cell 29(1):7-19.

[46] Xie, L., Pelz, C., Wang, W., Bashar, A., Varlamova, 0., Shadle, S., et al., 2011. KDM5B regulates embryonic stem cell self-renewal and represses cryptic intragenic transcription. The EMBO Journal 30(8):1473-1484.

[47] Ye, S., Li, P., Tong, C., Ying, Q.L., 2013. Embryonic stem cell self-renewal pathways converge on the transcription factor Tfcp2/1. The EMBO Journal 32(19):2548-2560.

[48] Shen, J., Jia, W., Yu, Y., Chen, J., Cao, X., Du, Y., et al., 2015. Pwp1 is required for the differentiation potential of mouse embryonic stem cells through regulating Stat3 signaling. Stem Cells 33(3):661-673.

[49] Valcárcel, R., Weber, U., Jackson, D.B., Benes, V., Ansorge, W., Bohmann, D., et al., 1999. Sec61beta, a subunit of the protein translocation channel, is required during Drosophila development. Journal of Cell Science 112(Pt 23): 4389-4396.

[50] Shaheen, R., Anazi, S., Ben-Omran, T., Seidahmed, M.Z., Caddle, L.B., Palmer, K., et al., 2016. Mutations in SMG9, encoding an essential component of nonsense-mediated decay machinery, cause a multiple congenital anomaly syndrome in humans and mice. The American Journal of Human Genetics 98(4):643-652.

[51] Katano-Toki, A., Satoh, T., Tomaru, T., Yoshino, S., Ishizuka, T., Ishii, S., et al., 2013. THRAP3 interacts with HELZ2 and plays a novel role in adipocyte differentiation. Molecular Endocrinology 27(5):769-780. 